\title{
Management of Vaccination Failure in a Case of HIV - HBV Co-infection: A Case Report
}

Andre Small, ${ }^{1}$ Hilary Schroeder, ${ }^{2}$ Raghu Maramraj, ${ }^{3}$ Marianinha Joanes. ${ }^{4}$

\begin{abstract}
Background: A 60-year-old African American female patient, with chronic HIV infection since 1999, presented with markers of acute hepatities B virus (HBV) infection for the past 15 months. The patient was previously vaccinated for HBV. Immunoglobulin dysfunction was hypothesized, but electrophoresis yielded no conclusive result. Results: Investigation suggests that the patient is a non-responder: someone who fails to sero-convert to standard vaccinations. This condition can be linked to B-cell dysfunction due to chronic HIV infection. Conclusion: It is suggested that non-responders may require a 6-dose regimen to achieve sero-conversion for vaccination. Prevention of co-infection should be the mainstay of treatment, which is achieved by vaccination. However, immune system dysfunction can lead to complications.
\end{abstract}

Keywords: HIV Seropositivity, Vaccination, Antigens, Hepatitis B (Source: MeSH, NLM).

\section{Introduction}

About the Author: Andre Small is currently in his fourth year of medical school, at the American University of Antigua. The school is located in St John's Antigua.
Submission: Dec 7, 2013 Acceptance: Jan 18, 2014 Process: Peer-reviewed
Human Immunodeficiency Virus (HIV) compromises the immune response by destroying cells paramount to the immune system, CD4 T-cells, eventually leading to morbidity and mortality from super-imposed infections, which commonly include Pneumocystis jiroveci, Cytomegalovirus and Toxoplasmosis. This makes prevention through vaccination and prophylaxis the cornerstone of HIV management. One such prophylaxis option is against the Hepatitis B virus (HBV). HIV-HBV co-infection is associated with dangerous consequences and as a result, prevention and/or management is extremely important. Despite antiretroviral therapy, around $10 \%$ of HIV patients who develop a super-imposed HBV co-infection develop liver disease. ${ }^{1}$ Patients with this type of co-infection are at increased risk of developing cirrhosis and fibrotic liver changes. ${ }^{2}$

HBV co-infection is common in many HIV infected patients at time of diagnosis, mainly due to the fact that both conditions are sexually and parentally transmitted viruses prevalent in people who engage in IV drug abuse and unsafe sexual practices. ${ }^{3}$ The use of highly active antiretroviral therapy (HAART) has led to increased survival, and subsequently increased prevalence of chronic viral hepatitis among people living with HIV. ${ }^{4}$ It is estimated that the prevalence of chronic co-infection is $8-11 \%$ in HIV patients..$^{5-8}$ As a result, it is routine to vaccinate all HIV patients for HBV. The Center for Disease Control (CDC) recommends that all high-risk patients receiving adult vaccinations, be given a 3 -dose vaccine series at 0,1 , and 6 months intramuscularly. ${ }^{9}$ However, many co-infected patients fail to seroconvert with standard vaccine administration they are deemed non-responders. Since co-infection has been attributed to

Key Points:
- HIV and HBV co-infection is a common occurrence amongst HIV patients.
This co-infection increases morbidity and mortality, and calls for particu-
lar treatment solutions.
- HIV patients can present with unique immune problems, such as B-cell
dysfunction.
- Viral infections and B-cell dysfunction can decrease the effectiveness
of standard vaccinations, therefore making patients non-responsive to
standard vaccinations.
- This articles discusses a case study of an HIV-HBV co-infected patient
who presents as a possible non-responder to standard HBV vaccination.
- We have suggested methods to deal with patients who present as
non-responders to standard HBV vaccinations.

a 19 -fold increase in mortality, ${ }^{10}$ it is important to establish a care plan for non-responders. This article discusses the clinical presentation of a non-responder and discusses the methods of management for such a patient.

\section{The Case}

A 60-year-old African American woman with chronic HIV infection presented to the clinic for a routine follow-up. She contracted HIV in 1999, and started treatment 4 months after the diagnosis. On her previous visit in January 2012, she showed serological evidence of an acute HBV infection, but with no clinical signs or symptoms. On that visit she was given treatment for HBV with raltegravir and HBV vaccine (Table 1). In April of 2013 the patient's blood tested positive for only HBC IgM antibody which was shown to be persistent for 15 months. Her $\mathrm{CD}_{4}$ count at this time was 947 . She still denied any symptoms such as weakness, fever, or right upper quadrant pain.

Her past medical history included a diagnosis of HIV in 1999 , hypertension in 1994, depression in 2000, hyperlipidemia, la-

\footnotetext{
${ }^{1}$ American University Of Antigua, St. John's, Antigua and Barbuda.

${ }^{2}$ International American University, CA, USA.

${ }^{3}$ Sint Eustatius School of Medicine, Cole Bay, Sint Maarten, the Kingdom of the Netherlands.

${ }^{4}$ Jackson Park Hospital, Chicago Il, USA. 
tent TB, HIV-related lipodystrophy syndrome, past history of gonorrhea, and an acute HBV infection. Her social history is significant for previous intravenous drug use she has been off drugs since the diagnosis of HIV. Her current anti-retroviral medications are atazanavir (Reyataz), ritonavir (Norvir) and raltegravir (Isentress). Medications to help control her hypertension are hydrochlorothiazide (HCTZ) and valsartan (Diovan). Finally, pravastatin (Pravachol) is used to control the hyperlipidemia. She has drug allergies to erythromycin and codeine.

On physical exam, the patient's vital signs were found to be within normal limits - meaning her temperature was $98.00 \mathrm{~F}$, pulse was 78 , respirations were 16 and her blood pressure was $110 / 70$. She was found to be moderately obese with a BMI of $32.3 \mathrm{~kg} / \mathrm{m}^{2}$ given her height of 65 inches and weight of 194 pounds. She did not seem to be in any obvious distress and showed signs of being normotensive and non-cachectic. Her head was normocephalic, pupils were equal and reactive, tympanic membranes were clear, mucosal surfaces were pink, and her throat was non-erythematous and free of exudates. Upon auscultation, her lungs were clear bilaterally, heart sounds were regular and there were not any murmurs or gallops and normal bowel sounds were heard in all four abdominal quadrants. Her abdomen was also soft, non-tender and there were not any signs of hepatosplenomegaly.

When the patient first presented in May 2012, her IgC and IgA were normal, and it was only her IgM that was subpar (1372, 246 and 31 , respectively). A complete blood count (CBC) was drawn 4 months later (September 2012) and revealed a WBC of 5.5, hemoglobin (Hgb) 12.9, hematocrit (Hct) 38 and platelets were 192,000. During the same visit, a lipid panel was ordered and the results showed a total cholesterol of 254, low-density lipoprotein (LDL) of 17, triglycerides (TC) of 159 and high-density lipoproteins (HDL) of 52 . Given her total cholesterol levels, the need to prescribe pravastatin was clear.

\section{Discussion}

This patient has a chronic HIV infection and presents with signs atypical of an acute HBV infection. Although numerous past attempts had been made to vaccinate this patient, she later presented with HBV after originally testing was negative. This patient classifies as a non-responder, due to failure to seroconvert, and produce IgG HBV surface antibodies. She has elevated levels of IgM core antibody, which typically presents during an acute infection. Furthermore, she has failed to produce IgG core antibodies or surface antibodies after 15 months of infection (Table 1). Thirty percent of viral hepatitis patients with associated drug use may present with isolated anti-HBc. These individuals tend to have a varied response to vaccination. ${ }^{1 "}$ It has been suggested that such patients may present with an occult liver disease. ${ }^{11}$ It was originally hypothesized that this particular patient may have had an IgG immunoglobulin deficiency. However, electrophoresis revealed the patient has IgC immunoglobulin the laboratory reference range.

Regardless of a typical or atypical presentation of HBV-HIV co-infection, a management plan is necessary. A review of literature has allowed advisement on suggested protocols to monitor and treat such patients.

Typically $\mathrm{CD}_{4}$ counts and HIV viral loads are used to monitor the disease process; however, B-Cells can also be affected in HIV ${ }^{6}$ HIV can affect and cause abnormalities in any lymphocyte group. Specifically, HIV can affect the frequency and composition of B-Cell subsets, especially in patients with chronic infection. ${ }^{12}$ The B-Cell dysfunction usually manifests as a decreased responsiveness to antigens, despite hypergammaglobulinemia and hyperactivity of the B-cells. ${ }^{13}$

In a 2012 study, vaccine responders and non-responders were followed after the diagnosis of HIV and subsequent vaccination. The goal of the study was to determine if non-responders were at an increased risk of mortality. It was concluded that hepatitis B surface antibodies could assist in the prediction of the progression to AIDS from HIV-infected patients. ${ }^{6}$ It was discovered that non-responders were associated with a 2 -fold increase in AIDS and/or death. It is hypothesized that HIV may play a role in the dysfunction of the interaction between the ligand of $\mathrm{CD}_{4}$ T-cells and B-Cells. ${ }^{6}$

HBV vaccination in HIV patients should be done while $\mathrm{CD}_{4}$ cell

Table 1. HIV Patient Labs Showing Resistance to Hepatitis B Vaccine Series.

\begin{tabular}{|c|c|}
\hline Vaccine/Test & Results [Date] \\
\hline Hepatitis Vaccine & [09/1999, 11/1999, 02/2000, 04/2012] \\
\hline Pneumovax & [2009] \\
\hline Fluvax & [10/2011] \\
\hline RPR & Negative [6/2011] \\
\hline Viral Load: HIV-1 & $<75$ undetectable $[01 / 2012],[5 / 25 / 2012]$ \\
\hline HCV SAb titer & Negative date: $[4 / 24 / 2013]$ \\
\hline Hepatitis screen & $\operatorname{HBC} \operatorname{IgM}(+) \mathrm{Ab}, \operatorname{HBsAg}(-), \operatorname{HBsAb}(-), \operatorname{HCV}(-)[5 / 25 / 2012],[4 / 24 / 2013]$ \\
\hline CD4 count & $796[01 / 2012], 880[5 / 25 / 2012], 947[4 / 24 / 2013]$ \\
\hline $\mathrm{CBC}$ & WBC 5.5, HCB 12.9, HCT 38, platelets $192,000[9 / 29 / 2012]$ \\
\hline Lipids & Chol 254, LDL 17, TC 159, HDL 52 [9/29/2012] \\
\hline Immunoglobulin C & $1372 \mathrm{mg} / \mathrm{dl}$ (ref range $694-1618$ ) $[5 / 25 / 2012]$ \\
\hline Immunoglobulin A & $246 \mathrm{mg} / \mathrm{dl}$ (ref range $81-463$ ) [5/25/2012] \\
\hline Immunoglobulin M & $31 \mathrm{mg} / \mathrm{dl}$ (ref range $48-271)[5 / 25 / 2012]$ \\
\hline
\end{tabular}


count is above 200, to achieve a higher probability of successful vaccination. Alternatively, patients who have a $\mathrm{CD}_{4}$ count below 200, should receive HAART first and then vaccinate when $\mathrm{CD}_{4}$ count is above 200.? Our patient has failed to seroconvert while having a $\mathrm{CD}_{4}$ count in the optimal range (Table 1).

Increased standard doses of hepatitis B virus vaccine, may prove to increase the results of seroconversion of non-responders. ${ }^{3}$ The current standard dose is three $20 \mu \mathrm{M}$ of vaccine given at 0,1 , and 6 months. ${ }^{9}$ In a study of 20 patients, it was discovered that non-responders receiving 6 doses had a seroconversion rate of $>90 \%$. The doses were given at 0,1 , and 2 months, followed by 3 additional doses at 3,4 , and 5 months. ${ }^{3}$

Co-infection of HBV and HIV requires special treatment. Co-infection can be treated with tenofovir. ${ }^{4}$ Lamivudine, has been used to treat these types of infections however, there are some infections that show resistance to lamivudine, thus allowing tenofovir to be used as an effective agent. The goal of therapy is to have a patient who is HIV-seronegative, with a manageable chronic hepatitis B state. Management should include monitoring liver histology, and prolongation of the time to development of end-stage liver disease. ${ }^{14}$

The most important management step of a HIV-HBV co-infection is prevention. Therefore, obtaining seroconversion should be the primary goal of medical treatment. Patients with a $\mathrm{CD}_{4}$ count below 200, should be treated with HAART in an effort to increase the $\mathrm{CD}_{4}$ count above 200 and increase the chances of seroconversion. In a patient with increased co-infection risk factors, such as continued intravenous drug use, utilization of a 6-doses vaccination method may be warranted. Alternatively, this method could also be used after failure of the standard vaccination regimen. The current literature emphasizes the importance of preventing co-infection. Furthermore, if a patient does develop co-infection, treatment with tenofovir should be administered as it has been shown to have a decreased rate of resistance.

Co-infection with HIV and HBV has an increased risk of morbidity and mortality. Prevention of co-infection should be the mainstay of treatment, which is achieved by vaccination. However, immune system dysfunction can lead to complications with vaccination, and result in vaccination non-responders. By knowing that B-cell dysfunction can lead to vaccine failure, a physician can better understand why alternative vaccine dosing regimens may be appropriate. In situations where co-infection is already present, treatment with an antiretroviral such as tenofovir should be used. The ultimate goal of management is to decrease viral serology, achieve a state where HIV viral load is undetectable, and delay the progression of end-stage liver disease.

\section{Acknowledgments}

None.

Conflict of Interest Statement ct Funding

The authors have no funding, financial relationships or conflicts of interest to disclose.

\section{Author Contributions}

Conception and design the work/idea: AS. Collect data/obtaining results: AS HS RM. Analysis and interpretation of data: AS HS MJ. Write the manuscript: AS HS MJ RM. Critical revision of the manuscript: AS HS MJ RM. Approval of the final version: AS HS MJ RM. Contribution of patients or study material: AS HS. Administrative or technical advice: AS HS.

Cite as:

Small A, Schroeder H, Maramraj R, Joanes M. Management of Vaccination Failure in a case of HIV - HBV Co-infection: A Case Report. Int J Med Students. 2014 Mar-Jun;2(2):68-70.

\section{References} long-term antiviral therapy in a North American cohort of hepatitis B virus (HBV)/human immunodeficiency virus type 1 (HIV-1) co-infected patients. J Clin Virol. 2013;57(2):103-8.

2. Thio CL, Seaberg EC, Skolasky R, Phair J, Visscher B, Muñoz A, et al. HIV-1, hepatitis $B$ virus, and risk of liver-related mortality in the Multicenter Cohort Study (MACS). Lancet. 2002;360(9349):1921-6.

3. Rey D, Krantz V, Partisani M, Schmitt MP, Meyer P, Libbrecht E, et al. Increasing the number of hepatitis $B$ vaccine injections augments anti-HBs 2000;18(13):1161-5. Efficacy of tenofovir disoproxil fumarate in antiretroviral therapy-naive and -experienced patients coinfected with HIV-1 and hepatitis B virus. J Infect Dis. 2004;189(7):1185-92.

5. Kellerman SE, Hanson DL, MCNaghten AD, Fleming PL. Prevalence of chronic hepatitis $B$ and incidence of acute hepatitis $B$ infection in human immunodeficiency virus-infected subjects. J Infect Dis. 2003;188(4):571-7.

6. Landrum ML, Hullsiek KH, O'Connell RJ, Chun HM, Ganesan A, Okulicz JF, et al. Hepatitis $B$ vaccine antibody response and the risk of clinical AIDS or death. PLOS ONE. 2012;7(3):e33488.

7. Lok AS, McMahon BJ. Chronic hepatitis B: update 2009. Hepatology. 2009;50(3):661-2.

8. Maek-a-Nantawat W, Avihingsanon A, Ohata PJ. Challenges in Providing Treatment and Care for Viral Hepatitis among Individuals Co-Infected with HIV in Resource-Limited Settings. AIDS Res Treat. 2012;2012:948059.

9. Mast EE, Margolis HS, Fiore AE, Brink EW, Goldstein ST, Wang SA, et al. A comprehensive immunization strategy to eliminate transmission of hepatitis B virus infection in the United States: recommendations of the Advisory Committee on Immunization Practices (ACIP) part 1: immunization of infants, children, and adolescents. MMWR Recomm Rep. 2005;54(RR-16):1-31.

10. De Vries-Sluijs TEMS, Hansen BE, van Doornum GJJ, Springeling T, Evertsz NM, de Man RA, et al. A prospective open study of the efficacy of high-dose recombinant hepatitis $B$ rechallenge vaccination in HIV-infected patients. Infect Dis. 2008;197(2):292-4.

11. Quaglio G, Lugoboni F, Mezzelani P, Des Jarlais DC, Lechi A. Hepatitis vaccination among drug users. Vaccine. 2006;24(15):2702-9.

12. Buckner CM, Kardava L, Moir S. Evaluation of B cell function in patients with HIV. Curr Protoc Immunol. 2013; Chapter 12:Unit 12.13

13. Moir S, Fauci AS. Pathogenic mechanisms of B-lymphocyte dysfunction in HIV disease. J Allergy Clin Immunol. 2008;122(1):12-9.

14. CDC - Centers for Disease Control and Prevention. Guidelines for the Prevention and Treatment of Opportunistic Infections Among HIV-Exposed and HIV-Infected Children. CDC - Morb Mortal Wkly Repor. 2009;58:1-176.
1. Coffin CS, Osiowy C, Myers RP, Gill MJ. Virology and clinical sequelae of response rate in HIV-infected patients. Effects on HIV-1 viral load. Vaccine.

4. Dore GJ, Cooper DA, Pozniak AL, Dejesus E, Zhong L, Miller MD, et al. 